\title{
Editorial
}

\section{From the Desk of the Section Editor (Abdominal Ultrasound)}

\author{
P.M. Venkatasai ${ }^{1}$ \\ ${ }^{1}$ Department of Radiology and Imaging Sciences, Sri Ramachandra \\ Medical College and Research Institute, Chennai, Tamil Nadu, India \\ J Gastrointestinal Abdominal Radiol ISGAR 2018;1:6
}

I am very proud to be a section editor of abdominal ultrasound for the Journal of Gastrointestinal and Abdominal Radiology ISGAR.

In my vast experience of more than 30 years in abdominal ultrasound, I have seen ultrasound, from gray scale to point of care, change clinical diagnosis to precise, nearpathological diagnosis. Enormous advances have revolutionized clinical confidence and vastly increased diagnostic accuracy. For example, elastography, microvascular imaging, and microbubble contrast agents have led to a paradigm shift in the practice of ultrasound. Ultrasound probes of different frequencies used for superficial to deep structures have improved the diagnostic accuracy of various conditions in abdominal ultrasound. Ultrasound-guided interventional procedures such as intussusception reduction, abscess drainage, cyst aspiration, and biopsies have a tremendous impact on patient management. Screening abdominal ultrasound can now display abnormalities early, thanks to superior resolution, and contributes to early detection, treatment, and, to some extent, prevention of disease.

I sincerely hope that the Indian Society of Gastrointestinal and Abdominal Radiology (ISGAR) and its journal will publish a host of exciting articles and educative material that will disseminate knowledge among clinicians and practicing radiologists alike. I thank all members of ISGAR, editorial board members of the journal, publishers, reviewers, and authors for their invaluable service to society.
Address for correspondence: P.M. Venkatasai, MBBS, DMRD, DNB, PhD, FICR, Department of Radiology and Imaging Sciences, Sri Ramachandra Medical College and Research Institute, Chennai 600116, Tamil Nadu, India (e-mail: hodvenkatasai@ gmail.com).
DOI https://doi.org/

10.1055/s-0039-1692874.
(C)2018 Indian Society of Gastrointestinal and Abdominal Radiology
License terms

(ㅇ) (1) $\ominus \circledast$ 